\title{
Incidence and socioeconomic cost of care to patients candidates to withdrawing life sustaining treatments in a Tunisian Intensive Care Unit
}

\author{
Nakhli M.S., Kahloul M., Chakroun A., Harrathi M.A., Chebbi N., Mhamdi S. \\ Sahloul Hospital, Dept of Anaesthesiology \& Intensive Care, Sousse, Tunisia
}

\section{Introduction:}

The absence of laws organizing the withdrawing life sustaining treatments (WLST) forces us to make unjustified therapeutic obstinacy. Our purpose is to determine the incidence of candidates to WLST decision, their sociodemographic characteristics and the socioeconomic cost of their medical care.

\section{Methods:}

This is a prospective study realized in the ICU of Sahloul teaching hospital during 3 months and including patients who could be candidates for WLST decision. Children, pregnant women and candidates for organ donations were excluded. The collected parameters were sociodemographic data, the reason for hospitalization, prognosis scales (Mccabe, Knaus, IGSII and SOFA), the arguments for WLST decision, cost of the medical care and medical evolution.

\section{Results:}

The number of patients who were candidates for WLST was eighteen (graphic 1) and their incidence was 10,4\%. The average age was 62,16 years with the existence of comorbidity in $83,3 \%$ of the cases (Table 1).

Table 1 : WLST candidate's reason for hospitalization

\begin{tabular}{llc}
\hline Reason for hospitalization & N & $\%$ \\
\hline Shock & 7 & 38,9 \\
\hline Septic Shock & 6 & 33,3 \\
Cardiogenic Shock & 1 & 5,6 \\
Traumatology & 5 & 27,8 \\
\hline With cervical trauma & 3 & 16,7 \\
Without cervical trauma & 2 & 11,1 \\
Stroke & 3 & 16,7 \\
Hemorrhagic stroke & 2 & 11,1 \\
Ischemic stroke & 1 & 5,6 \\
ARDS & 2 & 11,1 \\
Pancreatitis & 1 & 5,6 \\
\hline
\end{tabular}

The scales of Mccabe at 1 and knaus at $B$ were respectively noted in $55.6 \%$ and $44.4 \%$ of the cases. The IGSII and SOFA scales at the admission were respectively 59,39 and 10,83. The proposal of WSLT decision were made essentially by the medical expertise $(66,7 \%)$ with a timeframe of 5,33 days (Table 2). The length of stay was 21,5 days. The average cost of hospitalization from the proposal of WLST decision was 9724,4 TD (about 4006,5 euros) with a cumulated cost of 231635 DT (95436 euros). Only one patient survived but with severe sequelae.

Table 2 : Considered arguments in medical expertise

\begin{tabular}{lcc}
\hline & $\mathrm{N}$ & $\%$ \\
\hline Expected quality of life & 11 & 61,1 \\
Irreversibility of the acute disease & 10 & 55,5 \\
Lack of improvement & 7 & 38,9 \\
Severity of initial diagnosis & 6 & 33,3 \\
Multiple organ failure & 5 & 27,8 \\
Previous quality of life & 3 & 16,7 \\
Multiple medical histories & 3 & 16,7 \\
Underlying fatal disease & 1 & 5,6 \\
\hline
\end{tabular}

\section{References:}

\footnotetext{
1. Ferrand $\mathrm{E}$ et al. Réalité des pratiques de limitations et d'arrêts des soins en réanimation en France. Etude nationale. Réanimation (1997).

. Smedira NG et al. Withholding and withdrawal of life support from the critically ill. NEJM (1990).

Holzapfel $L$ et al. A four-step protocol for limitation of treatment in terminal care. An observational study in 475 intensive care unit patients. Intensive Care Med (2002).
}

Graphic 1 : study population

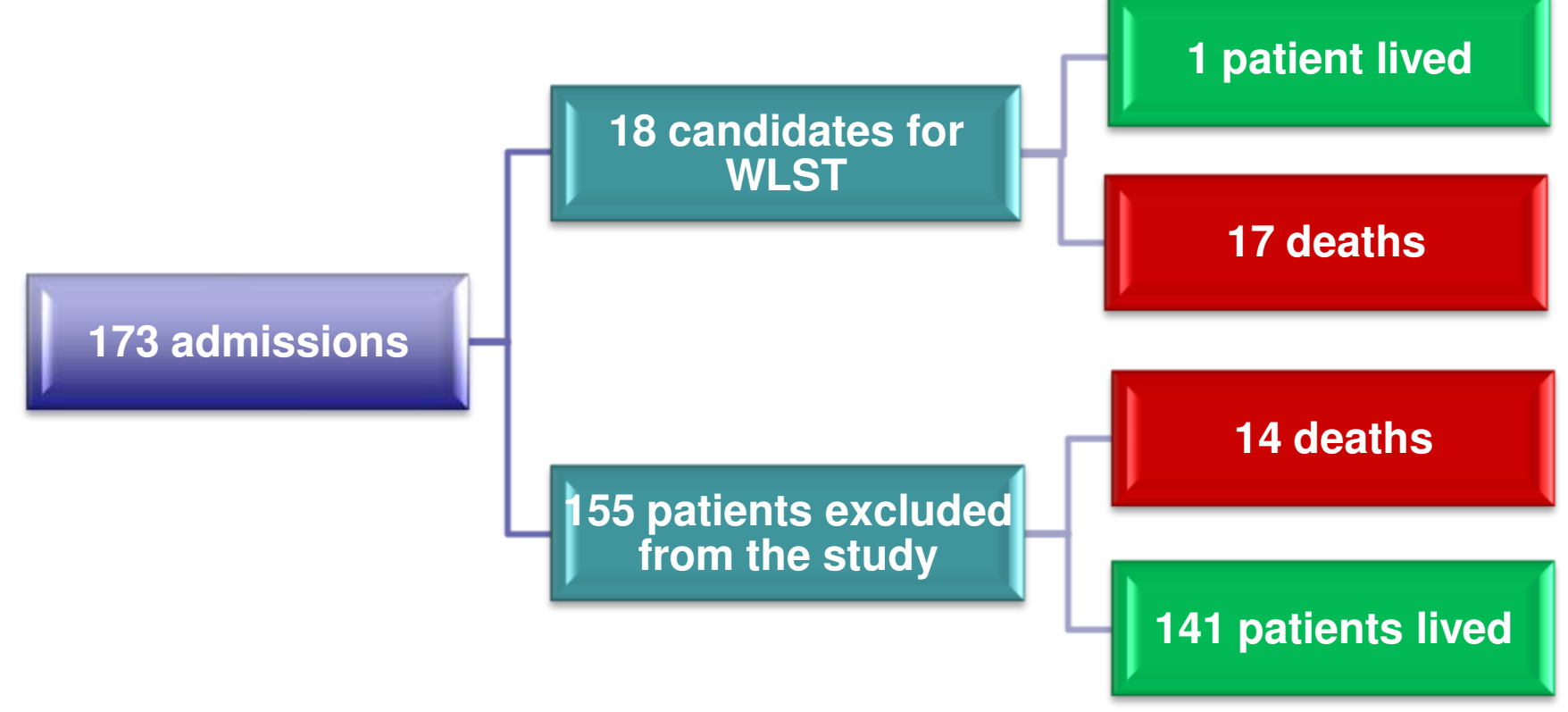

\section{Discussion:}

In our study, the incidence of the patients potentially candidate for WLS was $10.4 \%$. It was $9 \%$ for Robert and al, $11 \%$ in the LATAREA study (1), $7 \%$ for Smedira and al (2) and $17 \%$ for Holzapfel and al (3).

Our population was old so joining the literature's data. In an American study concerning 9000 ICU's patients, only $2 \%$ had an age $<50$ years, whereas $25 \%$ were more than 80 years old. In the study of Hakim and al, the decision not to resuscitate in case of ACR was 21 $\%$ for patients under 54 years old and of $55 \%$ for patients over 84 years old. According to a French study, the most frequent causes of hospitalization were the acute respiratory disorders (33\% of the cases) and the neurological disorders $(18,5 \%)$. In our study the identification of patients candidates for WLST were based essentially on the medical expertise. Certain validated scores such as KNAUS, MCCABE, IGSII and SOFA are of a considerable contribution because they eased the evaluation of comorbidities, previous quality of life and the gravity of the acute disease. In the literature, the current laws require the collegiality, the participation of the patient, his family or a reliable person and the participation of an Ethics Committee. Several departments have besides a protocol approach.

The global mortality was $17,91 \%$ in our department. The patients candidates for a decision of WLST represented $58 \%$. This mortality was $60 \%$ in the study of Robert, $53 \%$ in the study LATAREA (1) and $56 \%$ in the study of Montuclard.

In our work, the average cost of hospitalization from the proposal of WLST was 9724,4 DT (3700 euros) with a cost accumulated by 231635 DT (87 748 euros). According to the study CRRea, the total cost of a day of hospitalization was considered in euro $1425+/-101$ (3750 DT).

\section{Conclusion:}

The incidence of candidate patients to WLST is important with a considerable socioeconomic cost, justifying the necessity of a legal frame which organizes it in Tunisia. 